\title{
Erratum to: Normal-range albuminuria does not exclude nephropathy in diabetic children
}

\author{
Jacek Zachwieja • Jolanta Soltysiak • Piotr Fichna - Katarzyna Lipkowska • \\ Witold Stankiewicz • Bogda Skowronska • Pawel Kroll • \\ Maria Lewandowska-Stachowiak
}

Published online: 10 April 2010

(C) IPNA 2010

\section{Erratum to: Pediatr Nephrol}

\section{DOI 10.1007/s00467-010-1443-z}

It has come to our attention that the reported NGAL levels are 10-fold higher than those reported by other researchers. This is best seen from the normal values reported in Table 1. Inaccurate data is due to a decimal mistake. All reported serum and urine values for NGAL have to be divided by 10 .

The online version of the original article can be found at http://dx.doi. org/10.1007/s00467-010-1443-z.

J. Zachwieja $(\bowtie) \cdot$ J. Soltysiak $\cdot$ K. Lipkowska $\cdot$

M. Lewandowska-Stachowiak

Department of Pediatric Nephrology,

Poznan University of Medical Sciences,

Szpitalna 27/33 str.,

60-572 Poznan, Poland

e-mail: j.zachwieja@mp.pl

P. Fichna $\cdot$ W. Stankiewicz $\cdot$ B. Skowronska

Department of Pediatric Endocrinology and Diabetes,

Poznan University of Medical Sciences,

Poznan, Poland

P. Kroll

Department of Pediatric Urology,

Poznan University of Medical Sciences,

Poznan, Poland 